\title{
Prevalence of depression among Iranian patients with rheumatoid arthritis: a systematic review and meta-analysis
}

This article was published in the following Dove Medical Press journal: Open Access Rheumatology: Research and Reviews

\author{
Tayebeh Jamshidi' \\ Reza Ghanei Gheshlagh ${ }^{2,3}$ \\ Fariba Ebtekar ${ }^{4}$ \\ Sahar Dalvand ${ }^{5}$ \\ Amir Vahedian Azimi ${ }^{6}$ \\ Amanj Kurdi ${ }^{7}$
}

'Psychiatric Nursing Department, School of Nursing and Midwifery, Shahid Beheshti University of Medical Sciences, Tehran, Iran; ${ }^{2}$ Clinical Care Research Center, Kurdistan University of Medical Sciences, Sanandaj, Iran; ${ }^{3}$ Department of Nursing, Faculty of Nursing and Midwifery, Kurdistan University of Medical Sciences, Sanandaj, Iran; ${ }^{4}$ Department of Midwifery, Faculty of Nursing and Midwifery, Kurdistan University of Medical Sciences, Sanandaj, Iran; ${ }^{5}$ Department of epidemiology and Biostatistics, school of Public health, Tehran University of Medical sciences, Tehran, Iran; ${ }^{6}$ Trauma Research Center, Nursing Faculty, Baqiyatallah University of Medical Sciences, Tehran, Iran; ${ }^{7}$ Strathclyde Institute of Pharmacy and Biomedical Science, University of Strathclyde, Glasgow, UK

Correspondence: Reza Ghanei Gheshlagh Clinical Care Research Center, Kurdistan University of Medical Sciences, Pasdaran Ave, 66/8634683 Sanandaj 66/8634683, Iran

Tel+989l44050284

Email Ghanei@muk.ac.ir

\begin{abstract}
Patients with rheumatoid arthritis (RA) are prone to depression due to several factors related to their RA, including chronic and persistent pain, functional disability, economic constraints, and the side effects of RA medication. Previous Iranian studies showed conflicting and inconclusive findings regarding the prevalence of depression among RA patients. Therefore, this systematic review and meta-analysis was conducted to estimate the true prevalence of depression in Iranian patients with RA. Search for eligible articles was performed using the keywords of depression, depressive disorder, dysthymic disorder, major depressive disorder, RA, and Iran, and their possible combinations in the following databases: Scientific Information Database, MagIran, Web of Science/ISI, PubMed, and Scopus. The search was restricted to articles published in Persian and English languages. The meta-analysis was performed using the random effects model, and the data were analyzed using the STATA software version 12. Overall, six articles were selected; the overall prevalence of depression among the Iranian patients with RA was $65.58 \%$ (95\% CI: 56.53\%-74.62\%). There were no significant relationships between the prevalence of depression and articles' methodological quality and year of publication, participants' age, sample size, and duration of disease. More than half of RA patients suffer from depression. The overlap between the physical symptoms of RA and depression in this group of patients makes it difficult to correctly diagnose depression; therefore, initiative and efforts are required to improve the identification of early depression symptoms in RA patients in order to effectively manage their depression.
\end{abstract}

Keywords: depression, Iran, meta-analysis, prevalence, rheumatoid arthritis

\section{Introduction}

Rheumatoid arthritis (RA) is a systemic, chronic, and inflammatory disease that affects $0.5 \%-1 \%$ of the world's population. ${ }^{1}$ This painful and progressive disorder causes systemic disability and has no known treatment. Pharmacotherapies are only intended to reduce pain and inflammation, delay joint erosion, and maintain patient's normal functioning. ${ }^{2}$ While the etiology of the disease is unclear, genetic, infectious, hormonal, and environmental factors may play a role in RA. ${ }^{3}$ The unpredictable courses of the illness and pain affect all the aspects of the patient's life, such as social relationships, family life, and psychological well-being. ${ }^{4,5}$ Due to the different disease periods, patients with RA can hardly manage their daily life and disease process. ${ }^{5,6}$

Cohort studies have shown that the prevalence of depression is higher in patients with RA than in healthy subjects. ${ }^{7,8}$ Other cross-sectional studies have also shown that depression is more prevalent in patients with RA that in those with diabetes, cancer, or Parkinson's disease. ${ }^{4,6,9-11}$ 
Several factors that are related to RA could make this group of patients vulnerable to depression, including continuous pain, functional disability, inability to work, economic constraints, and the side effects of RA medication. ${ }^{11}$ Furthermore, depression in this group of patients may be caused by the interaction among clinical, biological, psychological, and demographic factors. ${ }^{10}$ Depression in these patients is associated with fatigue, reduction of quality of life, increased inability and costs of treatment, and reduced compliance with the therapeutic regimen. ${ }^{12-15}$ Depression not only affects the effective management of RA but also impacts the effective management of other comorbid conditions that are often associated with RA, such as cardiovascular diseases, infection, and cancer. ${ }^{16,17}$ These patients are always concerned about joint deformity, impaired functioning, and inability to do daily activities, and this constant worry can increase the likelihood of several negative outcomes, such as losing one's job, early retirement, and shorter working hours, hence, leading to a higher possibility of depression. ${ }^{3}$ Given the well-recognized, substantial negative impact of depression on patients' quality of life, adherence to therapeutic regimen, and its association with suicidal thoughts, early identification and effective treatment of depression in patients with RA is necessary. ${ }^{18}$ This requires accurate statistical description of the prevalence of RA as it would help in planning and developing methods for better diagnosis and management of depression. Therefore, this study was designed to estimate the prevalence of depression in Iranian patients with RA.

\section{Methods}

The screening and selection process was carried out in accordance with the PRISMA statement. ${ }^{19}$

\section{Search strategy}

In this systematic review and meta-analytic study, published articles in Persian and English languages in domestic and international journals were searched for in various databases (Scientific Information Database, MagIran, Web of Science/ ISI, PubMed [including Medline], and Scopus) from inception until February 2018. Search keywords were depression, depressive disorder, dysthymic disorder, major depressive disorder, RA, and Iran. The references of the identified articles were also accessed as the backtracking method to retrieve more articles.

\section{Selection of studies and data extraction}

All observational (noninterventional) articles reporting the prevalence of depression in Iranian patients with RA were eligible. The exclusion criteria included nonrelevant articles, case reports, interventional studies, repeated studies, and lack of access to the full text of articles. To reduce bias, the search process was performed independently by two researchers, and the disagreements between them were resolved through discussions. In the next step, the articles' information including the name of the first author, year of publication, place of study, sample size, type of instrument used, mean age of participants, duration of the disease, and the prevalence of depression were extracted and recorded on a specific form.

\section{Quality assessment}

The methodological quality of the selected articles was evaluated using a tool assessing five aspects: study plan, comparison groups, characteristics of the samples, sample size, and data collection tools. Each aspect was rated 0-3, and a higher score indicated a higher methodological quality $^{20-22}$ (Table S1).

\section{Statistical analyses}

Since the prevalence rate has a binomial distribution, the variance of prevalence was calculated using the binomial distribution variance formula. Also, the weighted mean was used to combine the prevalence rates reported by different studies. To evaluate the heterogeneity between the selected studies, the Cochran's $Q$ test and the $I^{2}$ statistic were used. Heterogeneity was classified into three categories of $<25 \%$ (low heterogeneity), 25\%-75\% (moderate heterogeneity), and over $75 \%$ (high heterogeneity). According to the level of heterogeneity between the selected studies $(89.3 \%)$ and the significance of $I^{2}$ statistic $(P<0.0001)$, the random effects model was used to combine the prevalence rates reported by the studies and estimate the total prevalence rate. The meta-regression was used to examine the relationship between the prevalence of depression with year of study, participants' mean age, duration of disease, and sample size. The subgroup analysis was used to estimate the prevalence of depression among RA patients in different regions of Iran. Egger's test and a Funnel plot were used to test the publication bias. The sensitivity analysis was performed through removing articles step by step. The data were analyzed using the STATA software v.12.

\section{Registration}

The study has been registered in the international prospective register of systematic review (PROSPERO) as number CRD42018100573. This study was a systematic review and meta-analysis of published studies, so it was not necessary to obtain an ethics approval or informed consent. 


\section{Results}

Of the 331 identified articles, a total of 6 articles were selected according to the inclusion criteria for the final analysis (Figure 1).

The overall sample size was 1,046 individuals within an average of 175 participants in each study. The largest and lowest sample sizes were for the studies of Jamshidi et al and Ghoreishi et al, respectively. ${ }^{23,24}$ The highest and lowest prevalence rates of depression were for the studies of Atapoor et al and Arman et al, respectively. ${ }^{25,26}$ In terms of methodological quality, all articles were in the moderate level. The general profile of selected studies for meta-analysis is reported in Table 1.

The overall prevalence of depression in patients with RA was 65.58 (95\% CI: 56.53\%-74.62\%) (Figure 2).

The prevalence of depression in patients with RA was 65.51\% (95\% CI: $54.88 \%-76.14 \%$ ) using the Beck Depression Inventory and $66.00 \%$ (95\% CI: 56.72\%-75.28\%) using the Hospital Anxiety and Depression Scale. The six final articles that had been screened and examined according to the PRISMA statement were assessed in terms of sensitivity. It was found that the overall estimate of the prevalence of depression was not changed by removing each of the studies. To investigate whether all articles on depression in Iranian patients with RA were included in the study, a bias diagram was drawn. Based on the Egger's regression test, publication bias was not significant ( $P=0.76$; Figure 3 ).

The results by the study language showed that the prevalence of depression in the Persian articles $(72.28 \%$; 95\% CI: $51.31 \%-93.25 \%$ ) was higher than those in the English articles $(62.09 \%$; 95\% CI: $52.60 \%-71.57 \%)$. The results of single-variable meta-analysis showed that the methodological quality of the articles $(P=0.88)$, participants' age $(P=0.77)$, sample size $(P=0.54)$, and duration of disease $(P=0.71)$ had no significant relationships with the prevalence of depression. An increase in the year of publication led to a downward trend in the prevalence of depression, which was not statistically significant ( $P=0.09$; Figure 4$)$.
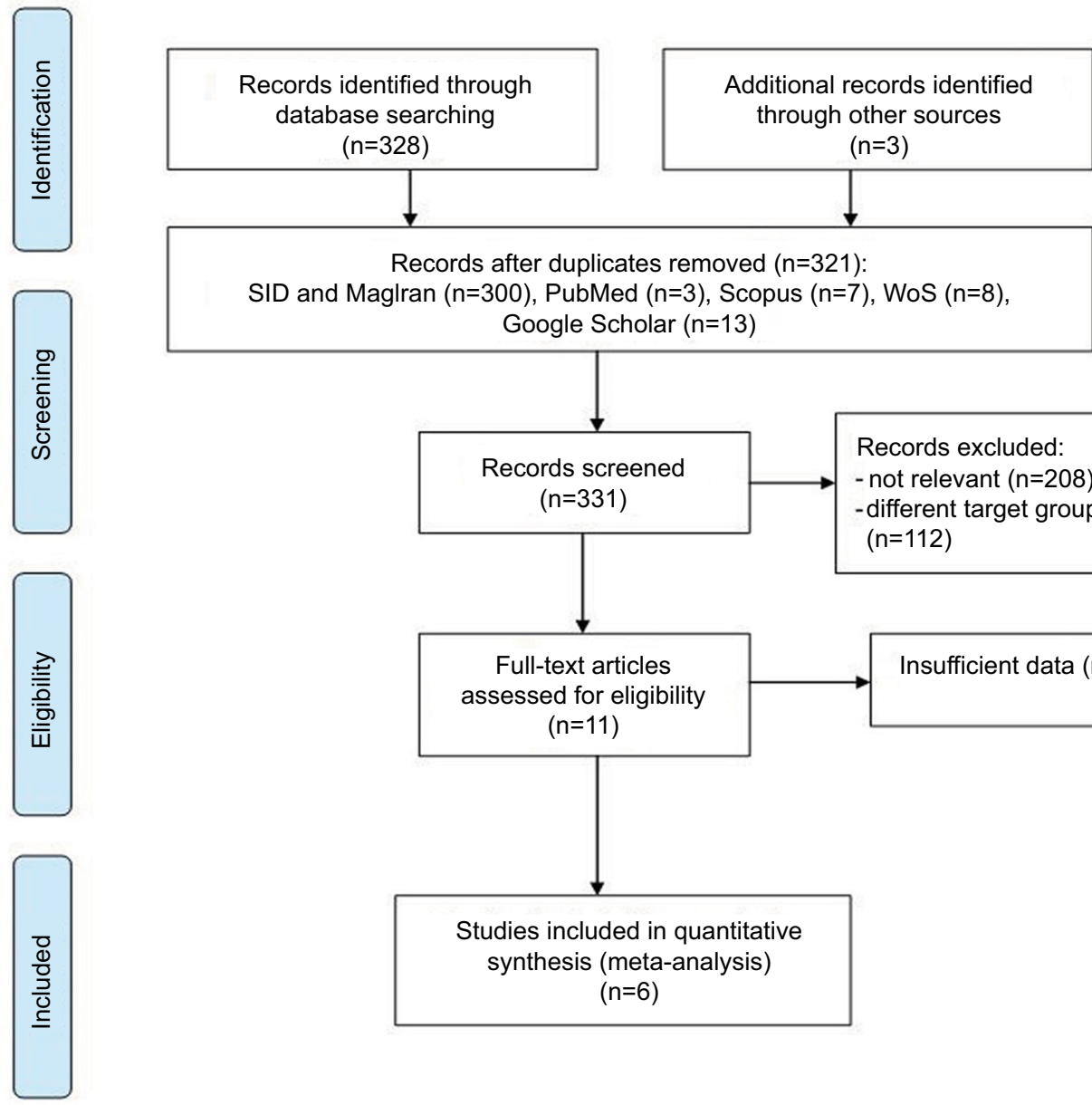

SID and Maglran $(n=300)$, PubMed $(n=3)$, Scopus $(n=7)$, WoS $(n=8)$, Google Scholar $(n=13)$

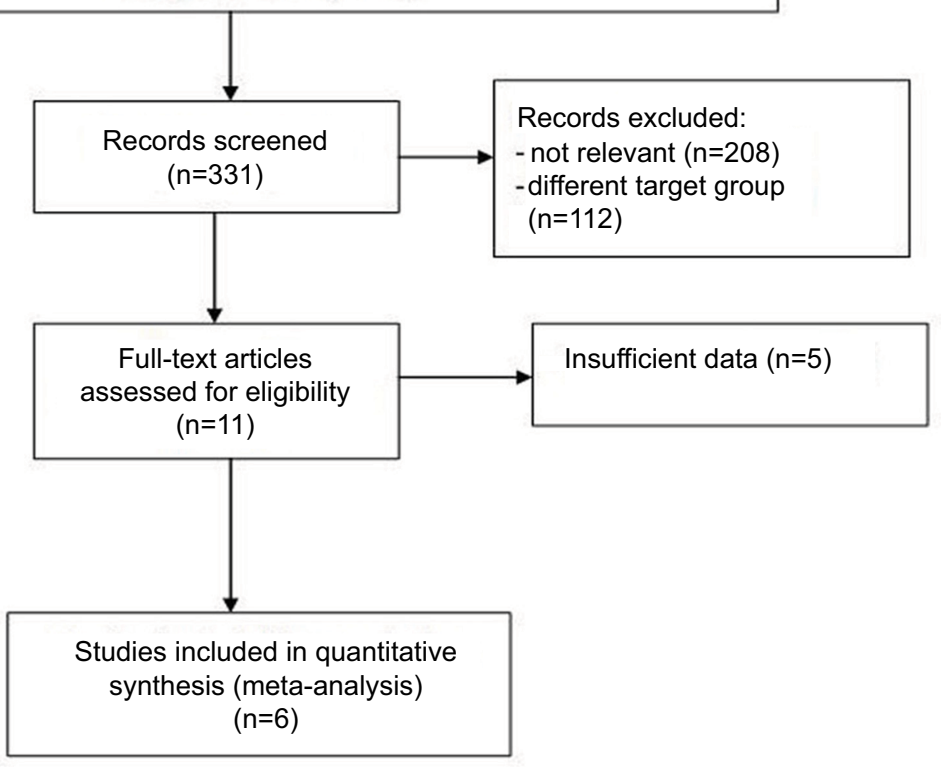

Figure I Screening process and selection of articles for meta-analysis according to the PRISMA statement. 
Table I Characteristics of the selected articles for meta-analysis

\begin{tabular}{|c|c|c|c|c|c|c|c|c|}
\hline First author & Year & $\begin{array}{l}\text { Sample } \\
\text { size }\end{array}$ & City & Tool & $\begin{array}{l}\text { Mean age, } \\
\text { years }\end{array}$ & $\begin{array}{l}\text { Length of } \\
\text { disease }\end{array}$ & $\begin{array}{l}\text { Quality } \\
\text { score }\end{array}$ & $\begin{array}{l}\text { Prevalence } \\
\text { (\%) }\end{array}$ \\
\hline Jamshidi et $\mathrm{a}^{23}$ & 2016 & 414 & Tehran & $\mathrm{BDI}$ & $45 \pm 11.6$ & $5.5 \pm 5$ & 5 & 63.6 \\
\hline Ghoreishi et $\mathrm{a}^{24}$ & 2016 & 81 & Kashan & $\mathrm{BDI}$ & - & - & 5 & 72.2 \\
\hline Arman et $\mathrm{al}^{26}$ & 2016 & 171 & Kermanshah & BDI & $45 \pm 11.2$ & - & 6 & 47.7 \\
\hline Rezaei et al' & 2014 & 100 & Isfahan & HADS & - & - & 7 & 66 \\
\hline Ghorbanshiroudi et al ${ }^{27}$ & 2013 & 180 & Rasht & $\mathrm{BDI}$ & $50.6 \pm 12.4$ & $8.6 \pm 6.4$ & 8 & 61.6 \\
\hline Atapoor et al ${ }^{25}$ & 2002 & 100 & Kerman & $\mathrm{BDI}$ & - & $6 \pm 6.5$ & 7 & 83 \\
\hline
\end{tabular}

Abbreviations: BDI, Beck Depression Inventory; HADS, Hospital Anxiety and Depression Scale.

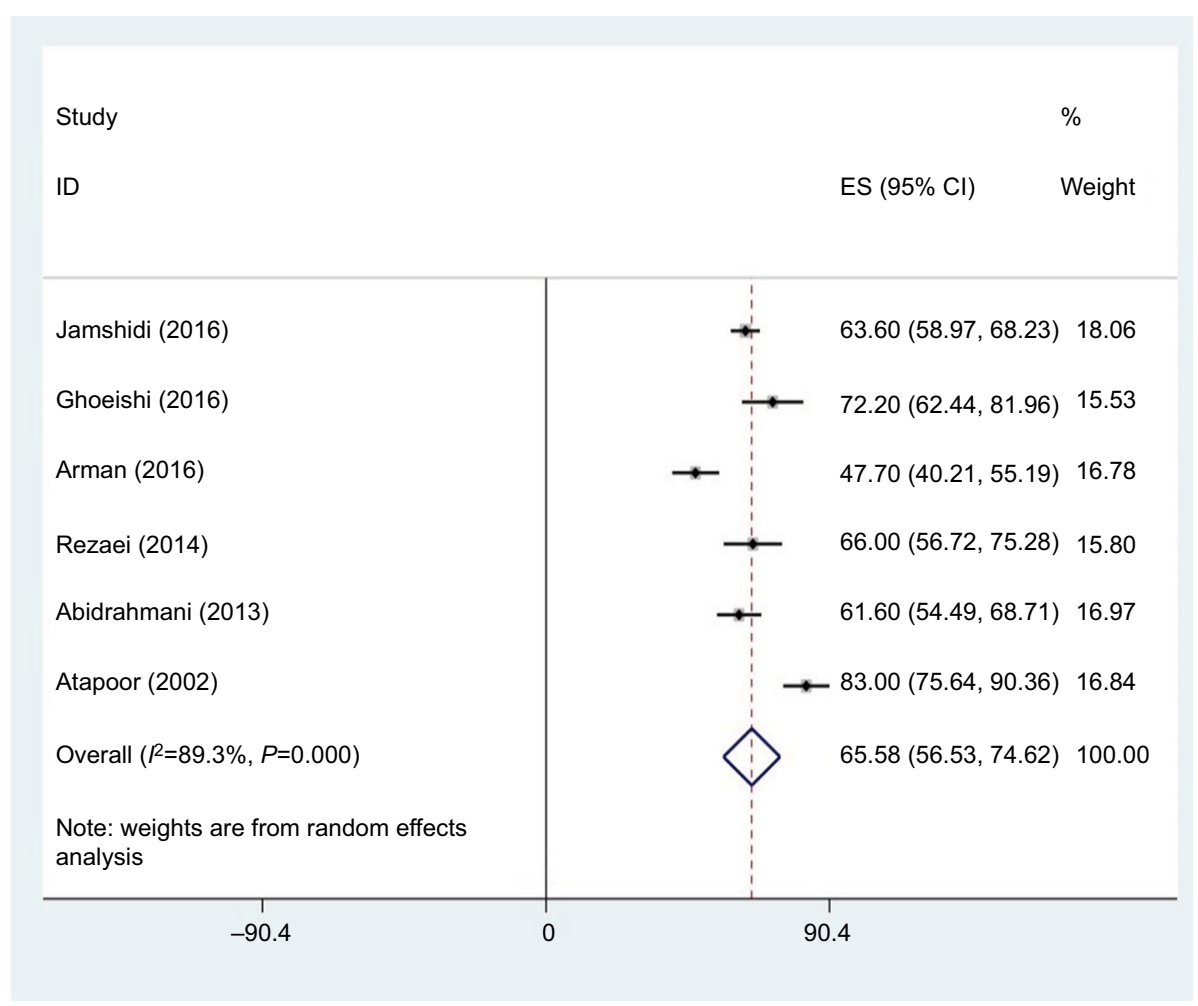

Figure 2 Overall prevalence of depressive symptoms among patients with rheumatoid arthritis.

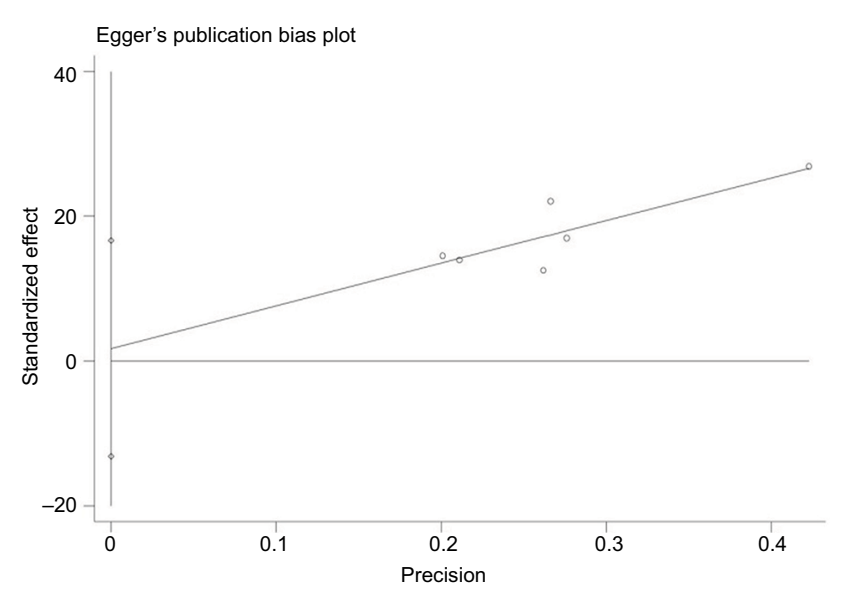

Figure 3 Publication bias in the selected studies. Note: The circles show the weight of each study.

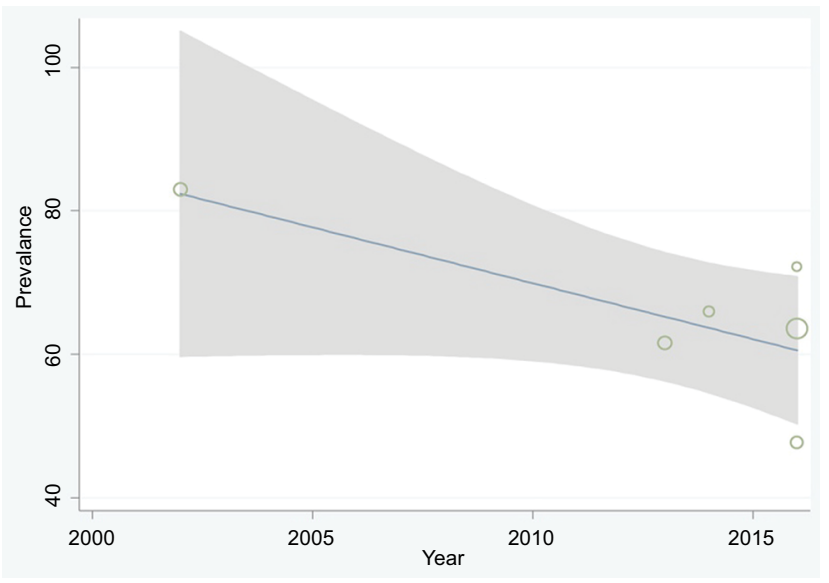

Figure 4 Prevalence of depression in patients with rheumatoid arthritis in terms of publication year.

Note: Circles show the weight of each study. 


\section{Discussion}

A review of the literature showed that $47 \%-83 \%$ of the Iranian patients with RA suffer from depression. These were different from the prevalence rates reported in other countries, such as $41.5 \%$ in Turkey and $71.5 \%$ in Pakistan. ${ }^{4,28}$ The study results also indicated that depression was more prevalent in patients with RA (65.5\%) than the other chronic diseases, such as hemodialysis (62\%), type II diabetes (54.8\%), and cardiovascular diseases $(47 \%)^{3,29-31}$

In this regard, it is important to note that in the Asian culture, depression is considered a kind of stigma, which is rarely expressed by patients explicitly; therefore, most cases are not diagnosed and treated. But in the Western culture, it is easy to describe this condition., ${ }^{7,32}$ Given the overlap between RA symptoms and depression, it may be difficult to diagnose depression in RA patients. ${ }^{33}$ Various studies suggested a high prevalence of depression in patients with RA. ${ }^{28-34}$ A meta-analysis of 12 studies showed the higher prevalence of depression in patients with RA than healthy subjects. ${ }^{35}$ Exposure to chronic mood disorders disrupts the hypothalamus-pituitary-adrenal axis, therefore, increasing the glucocorticoid release and the activity of the sympathetic system and decreasing the activity of the parasympathetic branches of the autonomic nervous system. Therefore, it ultimately causes changes in inflammatory parameters and the progression of autoimmune diseases. The results of a 14-year cohort study showed that the risk of depression in patients with RA was $70 \%$ higher than in healthy individuals. ${ }^{36} \mathrm{About}$ one-third of patients with RA lose their ability to work within 3 years of diagnosis and are incapable of doing their daily routine, which imposes a large financial burden on patients, families, and health care systems. ${ }^{7}$ Due to the inability to perform daily life activities and experiencing chronic pain, they may live in poor economic conditions that increases the possibility of experiencing depression. Another important factor for patients with RA is perceived social support. Patients with high levels of social support are less likely to have depression. ${ }^{37}$ According to the studies conducted in Iran, due to changes in cultural and social conditions, patients with chronic diseases may not have much social support, which can be associated with depression. ${ }^{38}$ Moreover, it has been reported that perceived social support was the most important predictor of depression and anxiety among patients with RA after controlling demographic variables. ${ }^{34}$

The results of the current study showed that there was no relationship between methodological quality, mean age of the participants, sample size, duration of the disease, and the prevalence of depression. With the increase of publication years, the prevalence of depression showed a downward trend, which was not significant. This can be attributed to medical advances in identifying and controlling the disease. Another finding was that there was an inverse association between the age of participants and the prevalence of depression in patients with RA. ${ }^{39}$ The prevalence of depression in the studies published in English was lower than that in the Persian articles; this may be attributed to the higher quality of English journals and the fact that the articles published in these journals undergo more in-depth evaluations.

Some of the articles had not reported their full results; therefore, the lack of accurate and comprehensive reports in some of the analyzed studies constituted one of the limitations of our meta-analysis. A comprehensive and up-to-date presentation of the prevalence of depression in patients with RA is one of the strengths of our meta-analysis. Overall, the findings of this study showed that more than half of patients with RA had depression symptoms. One of the most important limitations of this study was that some selected articles had not provided all the required information.

Given the overlap between the depression and RA symptoms, it is more difficult to diagnose depression in RA patients. Depression as an underlying illness, along with persistent pain, disability, and economic constraints, leads to a decrease in the patients' quality of life and reduces the patient's adherence to the therapeutic regimen. Informing health care providers and policy makers about the prevalence of depression in patients with RA can help design programs and initiate interventions useful in better diagnosis and effective management of depression in this group of patients. Future studies are suggested to use validated screening tools to diagnose those patients with RA who are at risk of developing depression, so that their condition can be controlled, and effective and holistic interventions can be provided for them.

\section{Acknowledgment}

This work was done without external funding.

\section{Disclosure}

The authors report no conflicts of interest in this work.

\section{References}

1. Rezaei F, Neshat Doost HT, Molavi H, Abedi MR, Karimifar M. Depression and pain in patients with rheumatoid arthritis: mediating role of illness perception. Egypt Rheumatol. 2014;36(2):57-64.

2. Matcham F, Ali S, Irving K, Hotopf M, Chalder T. Are depression and anxiety associated with disease activity in rheumatoid arthritis? A prospective study. BMC Musculoskelet Disord. 2016;17(1):155.

3. Iaquinta M, Mccrone S. An integrative review of correlates and predictors of depression in patients with rheumatoid arthritis. Arch Psychiatr Nurs. 2015;29(5):265-278. 
4. Isik A, Koca SS, Ozturk A, Mermi O. Anxiety and depression in patients with rheumatoid arthritis. Clin Rheumatol. 2007;26(6):872-878.

5. Bashar FR, Vahedian-Azimi A, Hajiesmaeili M, et al. Post-ICU psychological morbidity in very long ICU stay patients with ARDS and delirium. J Crit Care. 2018;43:88-94.

6. Soósová MS, Macejová Ž, Zamboriová M, Dimunová L. Anxiety and depression in Slovak patients with rheumatoid arthritis. J Ment Health. 2017;26(1):21-27.

7. Lin MC, Guo HR, Lu MC, Livneh H, Lai NS, Tsai TY. Increased risk of depression in patients with rheumatoid arthritis: a seven-year population-based cohort study. Clinics (Sao Paulo). 2015;70(2):91-96.

8. Vallerand IA, Lewinson RT, Frolkis AD, et al. Depression as a risk factor for the development of rheumatoid arthritis: a population-based cohort study. RMD Open. 2018;4(2):e000670.

9. Fu X, Li ZJ, Yang CJ, et al. The prevalence of depression in rheumatoid arthritis in China: a systematic review. Oncotarget. 2017;8(32):53623.

10. Ho RC, Fu EH, Chua AN, Cheak AA, Mak A. Clinical and psychosocial factors associated with depression and anxiety in Singaporean patients with rheumatoid arthritis. Int J Rheum Dis. 2011;14(1):37-47.

11. Mella LF, Bértolo MB, Dalgalarrondo P. Depressive symptoms in rheumatoid arthritis. Braz J Psychiatry. 2010;32(3):257-263.

12. van Hoogmoed D, Fransen J, Bleijenberg G, van Riel P. Physical and psychosocial correlates of severe fatigue in rheumatoid arthritis. Rheumatology. 2010;49(7):1294-1302.

13. Koop SM, ten Klooster PM, Vonkeman HE, Steunebrink LM, van de Laar MA. Neuropathic-like pain features and cross-sectional associations in rheumatoid arthritis. Arthritis Res Ther. 2015;17(1):237.

14. Margaretten M, Barton J, Julian L, et al. Socioeconomic determinants of disability and depression in patients with rheumatoid arthritis. Arthritis Care Res (Hoboken). 2011;63(2):240-246.

15. Kopciuch D, Paczkowska A, Leszczynsk P, Michalak M, Nowakowskai E. Effect of therapy with anti-TNF $\alpha$ drugs and DMARD on disease activity and health related quality of life among women with rheumatoid arthritis. Acta Pol Pharm. 2016;73(2):547-554.

16. Centers for Disease Control and Prevention [homepage on the Internet]. Rheumatoid arthritis; 2012. https://www.cdc.gov/arthritis/basics/rheumatoid-arthritis.html?CDC_AA_refVal=https $\% 3 \mathrm{~A} \% 2 \mathrm{~F} \% 2 \mathrm{Fwww} . c \mathrm{cc}$. gov\%2Farthritis\%2Fbasics\%2Frheumatoid.htm. Accessed 1 August, 2018.

17. Arthritis Foundation [homepage on the Internet]. Rheumatoid arthritis fact sheet; 2008. Available from: https://www.arthritis.org/aboutarthritis/types/rheumatoid-arthritis/. Accessed 1 August, 2018.

18. Tektonidou MG, Dasgupta A, Ward MM. Suicidal ideation among adults with arthritis: prevalence and subgroups at highest risk. Data from the 2007-2008 National Health and Nutrition Examination Survey. Arthritis Care Res (Hoboken). 2011;63(9):1322-1333.

19. Moher D, Shamseer L, Clarke M, et al. Preferred reporting items for systematic review and meta-analysis protocols (PRISMA-P) 2015 statement. Syst Rev. 2015;4(1):1.

20. Hoodin F, Weber S. A systematic review of psychosocial factors affecting survival after bone marrow transplantation. Psychosomatics. 2003;44(3):181-195.

21. Ghanei Gheshlagh R, Rezaei M, Ebadi A, Dalvandi A. Nourozi Tabrizi K. A systematic study of resilience in patients with chronic physical diseases. Nurs Midwifery Stud. 2017;6(2):e36401.

22. Ghanei Gheshlagh R, Nazari M, Baghi V, Dalvand S, Dalvandi A, Sayehmiri K. Underreporting of needlestick injuries among healthcare providers in Iran: a systematic review and meta-analysis. $J$ Hayat. 2017;23(3):201-213.
23. Jamshidi AR, Banihashemi AT, Paragomi P, Hasanzadeh M, Barghamdi M, Ghoroghi S. Anxiety and depression in rheumatoid arthritis: an epidemiologic survey and investigation of clinical correlates in Iranian population. Rheumatol Int. 2016;36(8):1119-1125.

24. Ghoreishi F, Zamani B, Razaghof M. Depressive disorder in rheumatoid arthritis: the more joint's distressed, the more severely depressed. Int $J$ Appl Behav Sci. 2016;3(1):31-35.

25. Atapoor J, Shakibi MR, Rajabizadeh G, Sarotehrigi M. The relationship between depression and disability in patient with rheumatoid arthritis in Kerman. J Kerman Univ Med Sci. 2002;9(2):79-85.

26. Arman F, Shakeri H, Jalilian F, Ebrahimi E, Shakeri J, Farnia V. The influence of pain, weakness and rheumatoid factor status on depression incidence among Iranian patients with rheumatoid arthritis. Iran J Psychiatry Behav Sci. 2016;10(3):e3894.

27. Ghorbanshiroudi S, Kalatbari J, Abid Rahmani A, Hajiabbasi A. Survey of depression and anxiety in patients with rheumatoid arthritis. J Guilan Univ Med Sci. 2013;22(85):15-22.

28. Khan EAS, Raja SF, Saeed MA, Haider II, Imran MY, Ahmad NM. Depression in rheumatoid arthritis and its relation to disease activity. Pak J Med Sci. 2015;31(2):393-397.

29. Ravaghi H, Behzadifar M, Behzadifar M, et al. Prevalence of depression in hemodialysis patients in Iran: a systematic review and meta-analysis. Iran J Kidney Dis. 2017;11(2):90-98.

30. Sheykhi A, Yadegarazadi A, Sayehmiri K, Rabiei Fakhr F, Azami M. The prevalence of depression in patients with type 2 diabetes in Iransystematic review and meta-analysis study. Iran J Diabetes Metab. 2016;15(4):217-224.

31. Ghaemmohamadi MS, Behzadifar M, Ghashghaee A, et al. Prevalence of depression in cardiovascular patients in Iran: a systematic review and meta-analysis from 2000 to 2017. J Affect Disord. 2018;227: 149-155.

32. Sruamsiri R, Kaneko Y, Mahlich J. The underrated prevalence of depression in Japanese patients with rheumatoid arthritis--evidence from a Nationwide survey in Japan. BMC Rheumatology. 2017;1(1):5.

33. Parker JC, Smarr KL, Slaughter JR, et al. Management of depression in rheumatoid arthritis: a combined pharmacologic and cognitivebehavioral approach. Arthritis Rheum. 2003;49(6):766-777.

34. Zyrianova Y, Kelly BD, Gallagher C, et al. Depression and anxiety in rheumatoid arthritis: the role of perceived social support. Irish J Med Sci. 2006;175(2):32-36.

35. Dickens C, McGowan L, Clark-Carter D, Creed F. Depression in rheumatoid arthritis: a systematic review of the literature with meta-analysis. Psychosom Med. 2002;64(1):52-60.

36. Lu MC, Guo HR, Lin MC, Livneh H, Lai NS, Tsai TY. Bidirectional associations between rheumatoid arthritis and depression: a nationwide longitudinal study. Sci Rep. 2016;6:20647.

37. Ratti MM, Rossi A, Delli Zotti GB, Sarno L, Spotti D. Social support, psychological distress and depression in hemodialysis patients. Psicologia Della Salute. 2017;1(2):112-122.

38. Faramarzi M, Hosseini S, Cumming R, et al. A negative association between social support and depression in the elderly population of Amirkola City. Br J Med Medic Res. 2015;8(8):707-716.

39. Matcham F, Rayner L, Steer S, Hotopf M. The prevalence of depression in rheumatoid arthritis: a systematic review and meta-analysis. Rheumatology (Oxford). 2013;52(12):2136-2148. 


\section{Supplementary material}

Table SI Criteria for rating the methodological quality of included articles

\begin{tabular}{|c|c|c|}
\hline Study parameters & Rating & Criteria \\
\hline \multirow[t]{4}{*}{ Study design } & 3 & Longitudinal prospective design \\
\hline & 2 & Retrospective or mixed design \\
\hline & 1 & Cross-sectional design \\
\hline & 0 & Survey or was not reported \\
\hline \multirow[t]{4}{*}{$\begin{array}{l}\text { Participants and } \\
\text { recruitment }\end{array}$} & 3 & $\begin{array}{l}\text { 1) Description of the population, 2) eligibility criteria for participants, 3) precise details of the recruitment } \\
\text { process, 4) accounted for the numbers recruited, and 5) lost to follow-up }\end{array}$ \\
\hline & 2 & Minimal description of at least four criteria \\
\hline & 1 & Two criteria missing \\
\hline & 0 & More than two criteria missing \\
\hline \multirow[t]{4}{*}{ Comparison group } & 3 & Healthy, age-appropriate comparison \\
\hline & 2 & Reference sample \\
\hline & 1 & Other comparison group \\
\hline & 0 & No comparison group \\
\hline \multirow[t]{4}{*}{ Number of participants } & 3 & $\mathrm{~N}>100$ \\
\hline & 2 & $\mathrm{~N}=50-100$ \\
\hline & 1 & $\mathrm{~N}<50$ \\
\hline & 0 & Did not report \\
\hline \multirow[t]{4}{*}{ Instruments } & 3 & Psychometrically sound report of generic or specific resilience measures \\
\hline & 2 & Psychometrically sound report of generic and specific resilience measures \\
\hline & 1 & Self-report generic or specific resilience measures with some psychometric data \\
\hline & 0 & Investigator constructed clinical rating of resilience or resilience domains with no psychometric properties \\
\hline
\end{tabular}

management of rheumatological diseases; Clinical trials and novel pharmacologi$\mathrm{cal}$ approaches for the treatment of rheumatological disorders. The manuscript management system is completely online and includes a very quick and fair peer-review system, which is all easy to use. Visit http://www.dovepress.com/ testimonials.php to read real quotes from published authors. 